\title{
MEMS MICRO-MECHANICAL LOGIC GATES FOR MECHANICAL COMPUTING IN MACHINE ONLY ENVIRONMENTS
}

\author{
Paul E. Kladitis \\ Air Force Institute of Technology (AFIT) \\ Department of Electrical and Computer Engineering \\ 2950 P Street \\ Wright-Patterson AFB OH 45433-7765 \\ Tel: 937-255-3636 ext. 4595, Fax: 937-656-4055, Email: paul.kladitis@afit.edu
}

\begin{abstract}
With respect to microsystems, environments such as harsh radiation, harsh temperature, or limited space may preclude the use of microelectronics and associated power supply for the purposes of sensor to actuator signal processing. For these situations, this work proposes the idea of performing the signal processing computation mechanically. To this end, this work presents novel MEMS micro-mechanical logic gates for mechanical computing. Micro-sized mechanical NAND, NOR, inverting logic gates, static memory cells, and optically readable outputs are developed, fabricated, and tested. A mechanical logic circuit, with the Exclusive-OR (XOR) function, is constructed from three mechanical NAND gates and demonstrated.
\end{abstract}

\section{INTRODUCTION}

In 1889, Charles Babbage invented his decimal based mechanical computer capable of all basic arithmetic operations including solving simultaneous equations, functions, and finding roots - example schematics are shown in Figure 1 [1]. Although Babbage's "Analytical Engine" was the size of a room, it probably had beneficial qualities such as being impervious to radiation and being able to operate at high temperatures. With the advent of Micro-Electro-Mechanical Systems (MEMS), machines can be created that are on the order of size of a human red blood cell. It now may be a worthwhile idea to design mechanical computers to take the place of equivalent functioning electrical computers in harsh environments where electronics will fail, such as radioactive or high temperature. Furthermore, there may be situations where the computation between microsensor and microactuator can be implemented mechanically, in a more convenient manner, than implementation by integrated electronics and power supply.

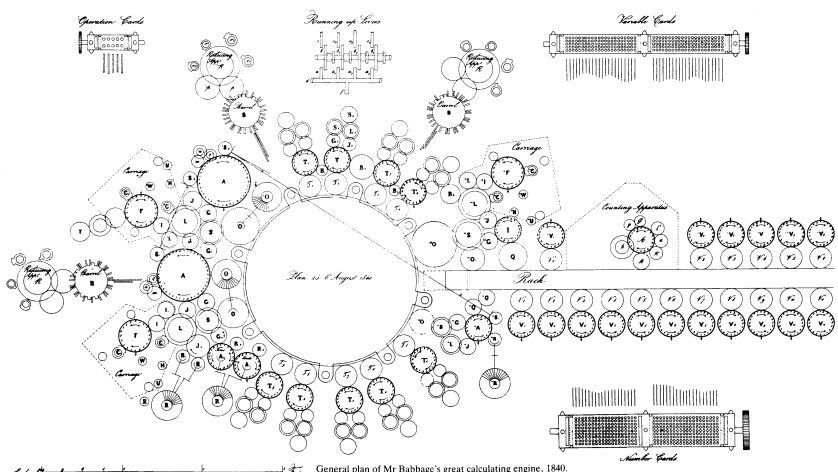

Figure1. Schematics of Babbage's “Analytical Engine” 1889 [1].

The art and science of mechanical computing has been around for a long time, as exemplified by Babbage's analytical engine or National Cash Register Co.'s (NCR) mechanical cash registers, Figure 2 [2]. In fact, mechanical computing is as ancient as mankind itself. Furthermore, electrical devices are just as ancient, and both have been used, where appropriate, throughout the ages Figure 3 shows pictures of a fossilized analog computer [3] complete with complex gearing, including differential gears, found in the Aegean Sea, and ancient batteries found near Baghdad [4], both dated as early as $1000 \mathrm{BC}$.

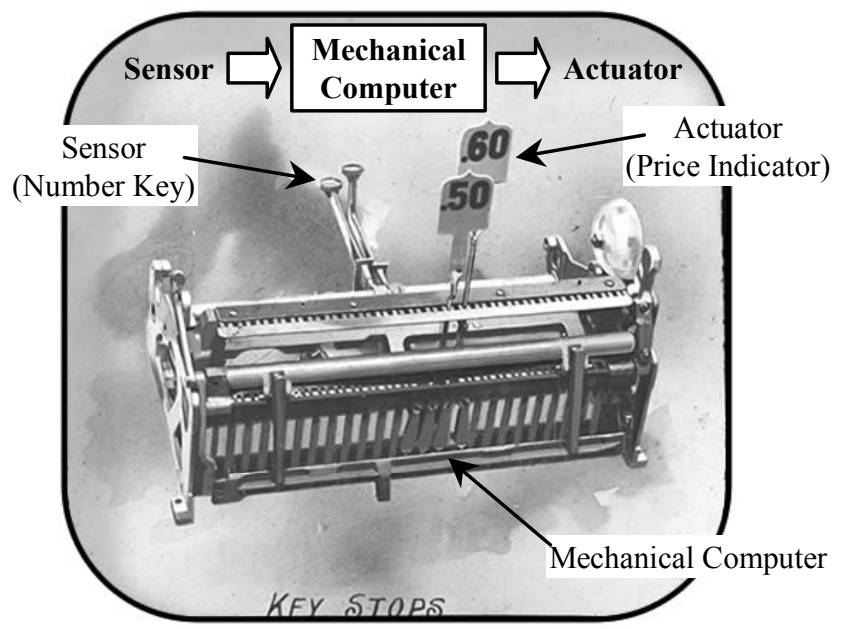

Figure 2. A "Magic Lantern" image of part of an 1891 NCR cash register [2].

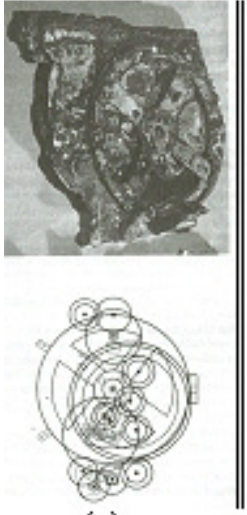

(a)

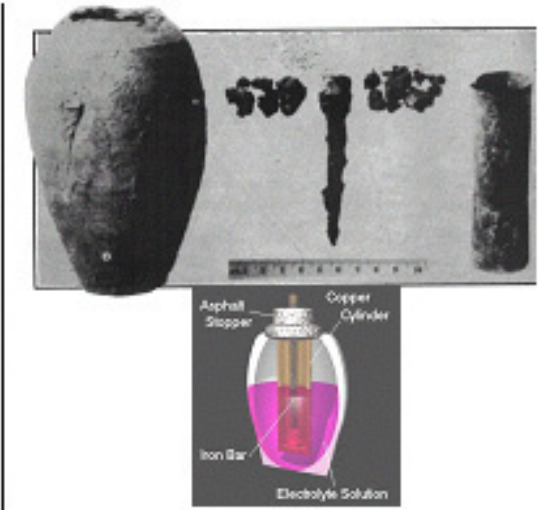

(b)
Figure 3. (a) Photograph of an analog computer including a sketch of $x$-ray photographs revealing complex gearing [3]. (b) Photograph of ancient battery and components including a depiction of a reconstructed battery [4], both dated at $\approx 1000 \mathrm{BC}$.

Mechanical computing can be categorized into analog computing and digital computing. This work demonstrates logic gates in mechanical form - a digital computing component. Electrical logic gates transform high and low electrical inputs into high or low electrical outputs. Mechanical logic (m-logic) gates transform motions at the inputs to motion at the output. This idea stands in contrast to the MEMS AND gate developed by Hirata et 
al. [5], where electrical signals are inputs and electrostatically actuated plates perform switching to provide electrical signal outputs. Similarly, the MEMS static RAM (SRAM) of Halg [6] also uses electrostatically actuated mechanical actuators to preserve an electrical state. Other work related to MEMS electromechanical digital computing can be found in [7-9]. This work deals with non-electrical mechanical movements only.

Mechanical logic functions are also not a new idea; some early examples include Lull's logic wheels [10]. Lull (1274 AD), a monk, designed turning wheels where the position of the wheel compared two ideas, where the ideas are abbreviated in Latin and printed on the wheel. Pastore's syllogism machine (1868) operates in two states: true if the wheels and belts all turn and false if the wheels can not turn [10], see Figure 4.

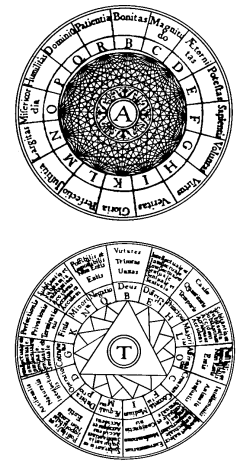

(a)

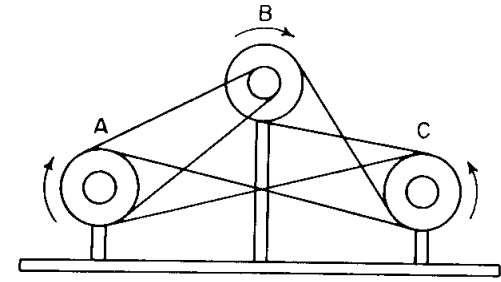

(b)
Figure 4. (a) Lull's logic wheels 1274, (b) Pastore's syllogism machine 1868 [10].

Presently, the idea of mechanical logic has been revived in the hopes of building super computers using atoms and molecules (nano-compting) [11 - 13]. Richard Feynman discussed the concept of atomic digital computing using "reversible logic gates," that is, gates where the output can be "undone." Given the output, the states at the input, that created the output in the first place, are recreated [14].

Although the m-logic gates in this work are not reversible, these gates may be, in some way, a step towards realizing nanocomputing systems of the future. Similarly, in 1943, the Navy's theoretical engineers (from MIT) proposed an all-electronic device, requiring 70,000 gas tubes, for decrypting the German's 4rotor Enigma Machine's coded messages, used by U-Boats in the Atlantic Ocean. However, Dayton, Ohio's Joseph R. Desch (1907 - Aug. 3, 1987), at NCR, realized that the gas tubes would generate too much heat to operate reliably. Desch chose to design and build the electro-mechanical NCR "Bombe," shown in Figure 5.

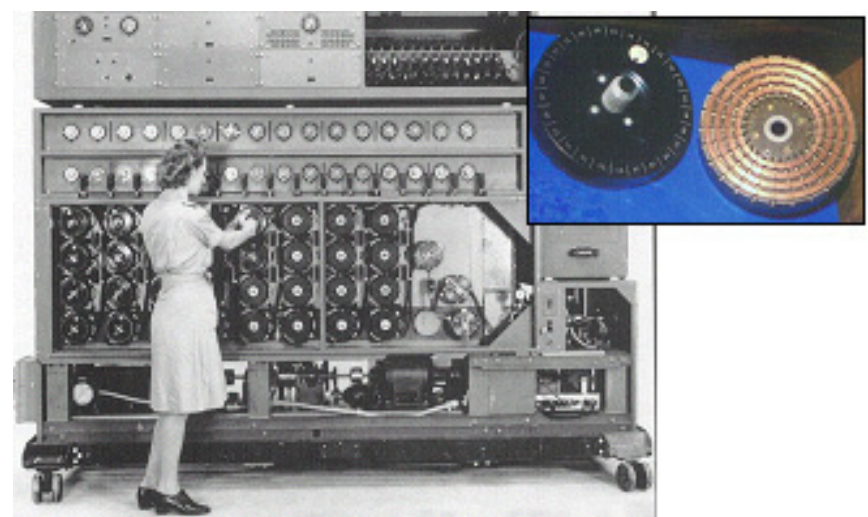

Figure 5. NSA Photograph of an NCR Bombe. A U.S. Navy WAVE (Women Accepted for Voluntary Emergency Service) is seen, possibly setting an initial position, on one of the rotors. Inset shows a close view of a rotor. Pictures courtesy of Jerry Proc.

The NCR Bombe "...helped win the battle for control of the Atlantic Ocean. During the war, this project was second only to the atom bomb in terms of national security and secrecy." [2] The NCR Bombe, since recent declassification, is now noted as an important step towards modern computing $[\mathbf{2}, \mathbf{1 5}, \mathbf{1 6}]$.

\section{THEORY AND DESIGN}

In this research, micro-sized mechanical NAND (m-NAND), NOR (m-NOR), inverting logic gates (m-inverter), static memory cells (m-RAM), and optically readable outputs are developed, fabricated, and tested. Figure 6 depicts an m-NAND gate in its four different states - states also indicated in the inset "truth table".
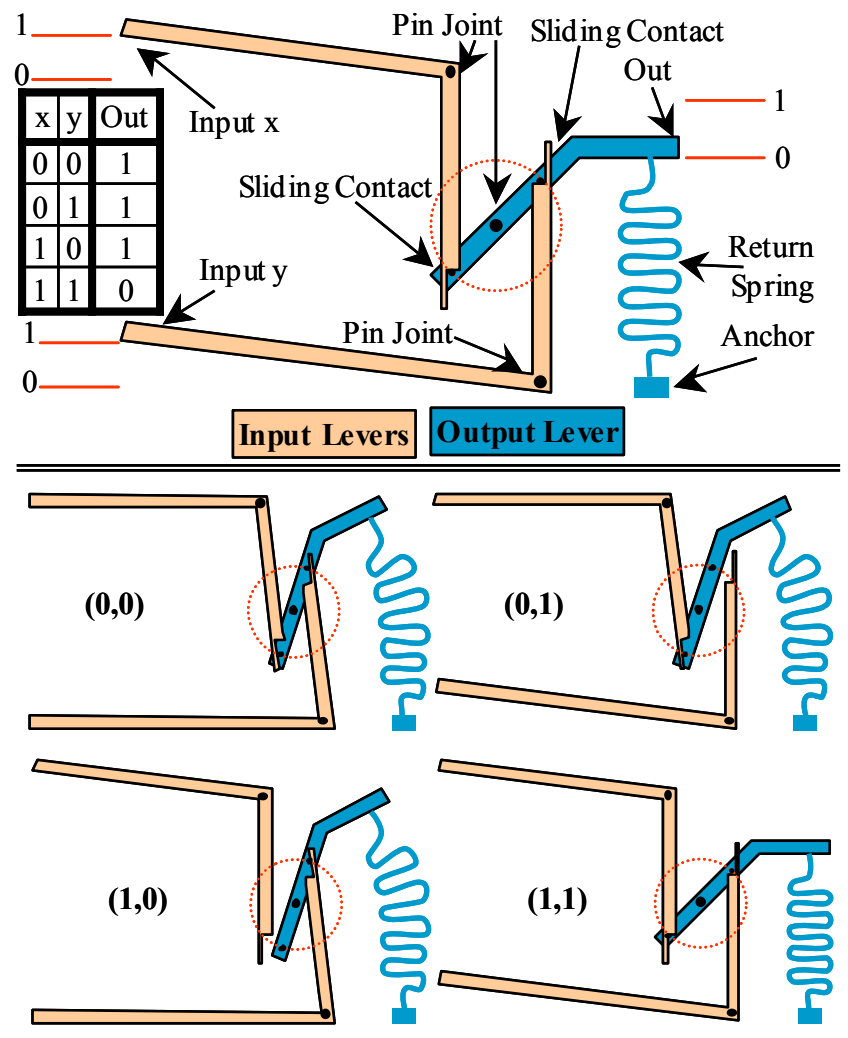

Figure 6. Depiction of an m-NAND gate, a NAND "truth table," and depictions of the m-NAND's four different states $(x, y)$.

The design of the m-logic circuits has a one-to-one correspondence with the existing design methodology of electronic combinational and sequential digital logic circuits. The only difference in design concept is as follows: an electronic logic gate requires either $0 \mathrm{~V}$ or $5 \mathrm{~V}$ inputs and provides a logical $0 \mathrm{~V}$ or $5 \mathrm{~V}$ output; the m-logic gate's inputs are levers that require either "down" or "up," and the output lever provides a logical "down" or "up". The Boolean algebra, used in design, is still the same, where $0=$ "down" and $1=$ "up".

The simplest $\mathrm{m}$-logic gate, the m-inverter, is simply a lever. The m-inverter is depicted in Figure 7. An m-inverter can also be created by physically connecting the inputs of the m-NAND together as one input.

The output of an m-logic circuit can be used as actuation or can be read optically. Furthermore, the state of an output can be preserved, in effect, making static memory (m-SRAM). However, a mechanism to disconnect the input of the m-SRAM from the rest of the circuit, until a memory update occurs, may be necessary. 
Figure 8 shows the concept of mechanical m-SRAM and optical output where the output (a mirror) is optically covered ("down") or uncovered ("up"). For the memory, a simple spring lever can be used to preserve a "down" or "up" state until forced to change otherwise. The m-SRAM is not demonstrated in this work.

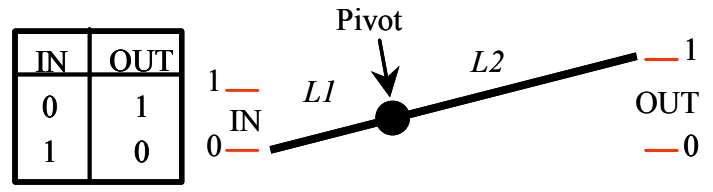

Figure 7. Depiction of an m-inverter where $L 1$ is the distance from the tip of the input to the pivot, and L2 is the distance from the tip of the output to the pivot.

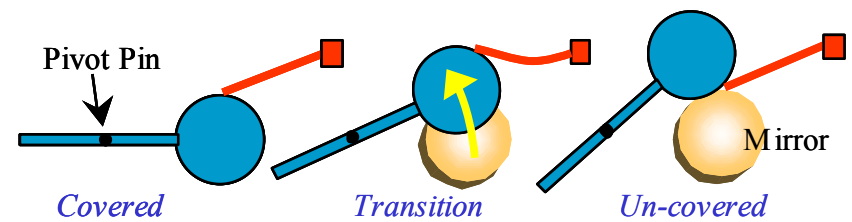

Figure 8. Depiction of m-SRAM and optical output.

\section{FABRICATION AND EXPERIMENTAL DETAILS}

Figure 9 (a) shows a scanning electron micrograph (SEM) of a fabricated and released m-NAND, and (b) shows an m-NOR. The sliding contacts are implemented in a slightly different manner than depicted in Figure 6. Figure 10 shows a captured video image of an m-NOR with optical output.
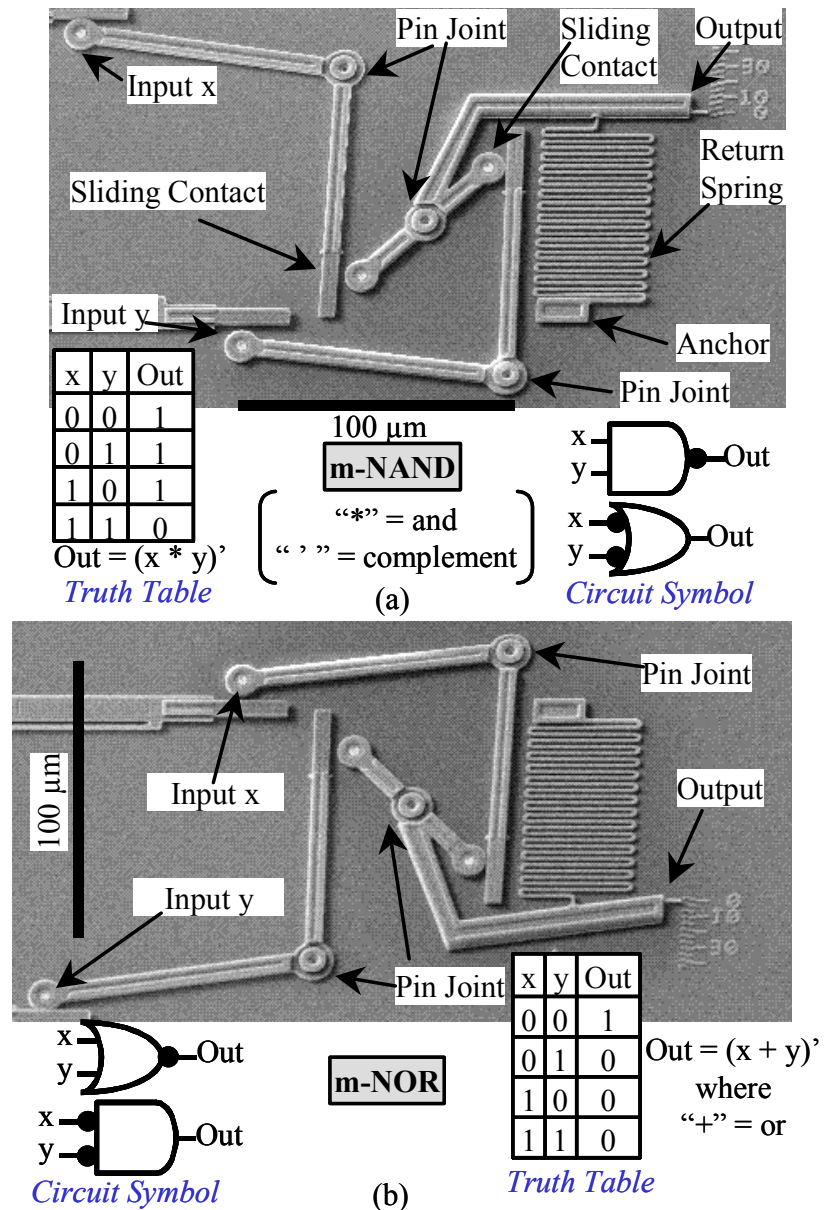

Figure 9. SEM of a fabricated and released (a) $m-N A N D$ and (b) $m-N O R$, and respective truth tables and circuit symbols.

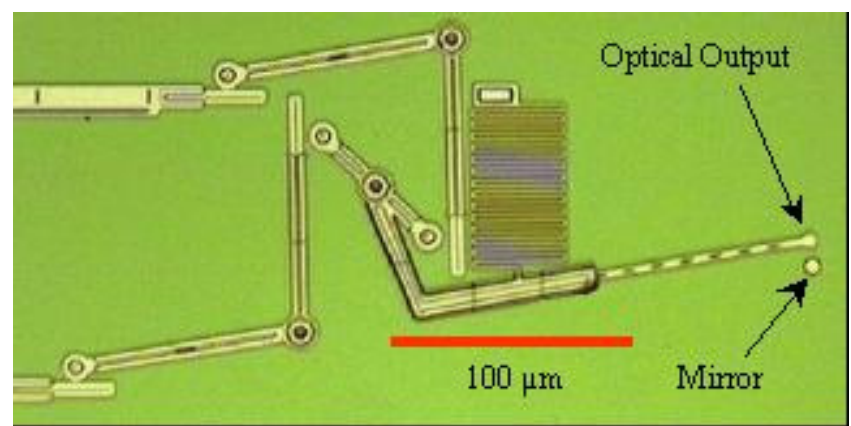

Figure 10. Video image of an m-NOR with optical output.

The mechanical logic circuits were fabricated using the MUMPs polysilicon surface micromachining process [17], which has two releasable polycrystalline silicon structural layers: poly1 and poly2, poly2 being the upper layer. The input and output levers are made from a stacked combination of poly1 and poly2. The pin caps of the pin joints are made from poly2. The return spring is made from poly1. Each link, of the 28-link return-spring, is $66 \mu \mathrm{m}$ long and has a cross sectional area of $2 \mu \mathrm{m} \times 2 \mu \mathrm{m}$.

The position of the return-spring connection to the output lever, and length of the output lever, can be varied to achieve a required input actuation force for a desired output deflection, respectively. The following discussion assumes an arbitrary output lever deflection (at the return-spring attachment) of $10 \mu \mathrm{m}$. The reaction force of 16- to 21-link springs, stretched $10 \mu \mathrm{m}$, was modeled using finite element analysis (FEA). The force required to stretch the 16-link spring was predicted to be $6.94 \mu \mathrm{N}$. From beam theory approximations of the spring, corroborated by FEA, the force required $(F)$ to stretch an $n$-link spring is $F=6.94 \mu \mathrm{N} \times$ $16 / n$. Therefore, the restoring force of the 28 link spring when stretched $10 \mu \mathrm{m}$, for the m-gates depicted in Figures 9-11, is approximately $6.94 \mu \mathrm{N} \times 16 / 28=3.97 \mu \mathrm{N}$. Since the lever arm of the spring attachment point is twice as long as the input force lever arm, the force required at a single input, of the m-logic gates, is approximately $2 \times 3.97 \mu \mathrm{N}=7.94 \mu \mathrm{N}$. The input lever distance required to deflect the spring attachment point by $10 \mu \mathrm{m}$ is $10 \mu \mathrm{m} /$ $2=5 \mu \mathrm{m}$.

The m-logic circuits were released and tested by mechanically manipulating the inputs with probes and observing the outputs under a microscope. Figure 11 shows a sequence of captured video images of a mechanical Exclusive-OR (m-XOR) circuit composed of 3 m-NANDs - just as the electronic XOR would be. The $\mathrm{m}-\mathrm{XOR}$ in Figure 11 (a) is in a nonsense state since inputs $\mathrm{x}$ ' and y' are not set appropriately. In Figure 11 (b) - (e), the m-XOR inputs are appropriately sequenced through the four input states by deflecting the input levers with probes. Down arrows indicate deflected (zero/down state) inputs in Figure 11 (b) - (e).

\section{DISCUSSION}

The m-logic circuits suffer from displacement fan-out and force fan-out. Force fan-out is where the output force diminishes due to increasing levels of logic gates thereby increasing pivot and sliding contact friction points and the number of return springs that the input force must overcome. Displacement fan-out is where the output displacement diminishes with increasing number of logic gate levels. Displacement fan-out occurs because the m-gates are surface micromachined, and due to the fabrication process, must have a finite space between the sliding contacts and at the pin joint. These finite spaces add up with each level of logic gates. The minverter can be used to compensate for either type of fan-out, but not both. The output displacement is $L 2 / L 1$ times the input displacement, while output force is $L 1 / L 2$ times the input force. 
The dotted-line circles, in Figure 11 (a), indicate the location of the interface sliding contacts between the first level of $\mathrm{m}$ NANDs and the second level output m-NAND. Before release, the output levers of the first level of m-NANDs are initially located below the inputs to the second level m-NAND. To prime the circuit for proper operation, the output levers of the first level of m-NANDs have to be manually (using micromanipulators) lifted to the other side of the second level m-NAND inputs as shown in Figure 11.

Plans for future work includes the following: investigate solutions for optimizing gate design by reducing fan-out and gate size; develop logic level interconnections that do not require manual assembly; demonstrate analog mechanical computing components such as differentiators, integrators, and ALUs; fabrication mechanical computing circuits in $\mathrm{SiC}$; and confirm operation of $\mathrm{SiC}$ and polysilicon mechanical computing circuits while in radiation and high temperature harsh environments.

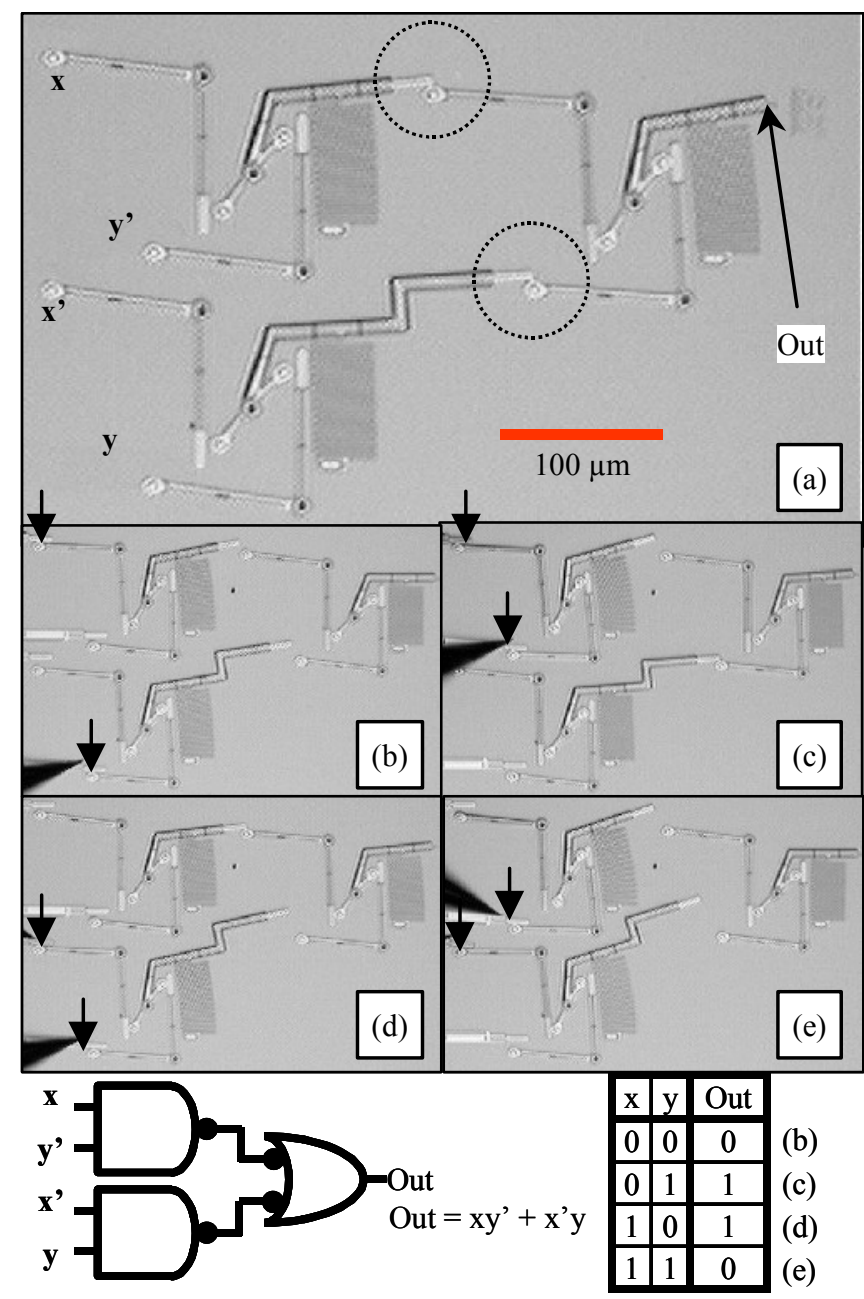

Figure11. Captured video images of an $m$-XOR circuit composed of $3 m$-NANDs. The states (b) - (e) of the $m$-XOR are indicated to the right of the truth table. Arrows indicate inputs being deflected.

\section{CONCLUSIONS}

This work presented an overview of mechanical computing and the design and implementation of m-NANDs, m-NORs, optical output, and the implementation of an $\mathrm{m}$-XOR. The concept of the m-inverter and m-SRAM were presented. M-logic gate shortcomings and future work were presented in the Discussion section. Micro m-logic circuits provide a complete non-electronic paradigm for logical computing and may serve as an alternative to radiation hard electronics or electrical sensors and control circuits for high temperature applications. Furthermore, simple mechanical computing, instead of electronic control, between sensor and actuator on a micro platform (robot, satellite, UAV, thruster system), may save space, energy, and be a simpler and straightforward means of implementing control.

\section{ACKNOWLEDGMENTS}

The mechanical logic gate idea was conceived, fabricated, and demonstrated by the author, in 1999, while working on a Doctor of Philosophy degree at the University of Colorado at Boulder. Dr. Victor Bright and Dr. Y. C. Lee provided funding for the fabrication. Thanks!

\section{REFERENCES}

[1] M. Campbell-Kelly, The Works of Charles Babbage, Pickering \& Chatto, London, 1989.

[2] The National Cash Register Co. (NCR) archives of The Montgomery County Historical Society, 224 North St. Clair Street, Dayton, Ohio 45402, Phone: 937-228-6271, Fax: 937-331-7160, E-mail: MCHS@Daytonhistory.org.

[3] D. Chittick, The Puzzle of Ancient Man, Creation Compass, 1997.

[4] P. T. Keyser, "The Purpose of the Parthian Galvanic Cells," Journal of Near Eastern Studies, vol. 52, no. 2, p. 98, 1993.

[5] A. Hirata, K. Machida, H. Kyuragi, and M. Maeda, "A Micromechanical Switch as the Logic Elements for Circuits in Multi Chip Module on Si (MCM-Si)," Proceedings of the 12th IEEE International Conference on MEMS, pp. 582-587, 1999.

[6] B. Halg, "On a Nonvolatile Memory Cell Based on MicroElectro-Mechanics," Proceedings of the IEEE Conference on MEMS, pp. 172-176, 1990.

[7] E. J. J. Kruglick and K. S. J. Pister, "MEMS Relay Based Digital Logic Systems," $10^{\text {th }}$ International Conference on SolidState Sensors and Actuators: Transducers'99, pp. 818-821, 1999.

[8] R. Yeh, R. A. Conant, and K. S. J. Pister, "Mechanical Digital-to-Analog Converters," International Conference on SolidState Sensors and Actuators: Transducers'99, pp. 998-1001, 1999. [9] H. Toshiyoshi, D. Kobayashi, M. Mita, G. Hashiguchi, H. Fujita, J. Endo, and Y. Wada, "Micro Electro Mechanical Digitalto-Analog Converter," $10^{\text {th }}$ International Conference on SolidState Sensors and Actuators: Transducers'99, pp. 994-997, 1999.

[10] M. Gardner, Logic machines and diagrams, McGraw-Hill, 1958.

[11] E. Fredkin and T. Toffoli, "Conservative Logic," International Journal of Theoretical Physics, vol. 21, nos. 3/4, pp. 219-253, 1982.

[12] E. K. Drexler, Nanosystems, John Wiley \& Sons, 1992.

[13] R. C. Merkle, "Two types of mechanical logic," Nanotechnology, vol. 4, pp. 114-131, 1993.

[14] R. Feynman, "Infinitesimal Machinery (transcription of 1983 speech)," Journal of Microelectromechanical Systems, vol. 2, no. 1, pp. 4-14, March 1993.

[15] J. DeBrosse, "Enigma," The Dayton Daily News, An 8 part series beginning February 25, 2001.

[16] Center for Cryptologic History, "The Bombe: prelude to modern cryptanalysis," National Security Agency (NSA), Fort George G. Meade, MD, OCLC number: 35657417, 1990.

[17] D. A. Koester, R. Mahadevan, A. Shishkoff, and K. W. Markus, MUMPs ${ }^{\mathrm{TM}}$ Design Handbook, Revision 4.0, Cronos Integrated Microsystems, 3021 Cornwallis Road, Research Triangle Park, NC 27709, May 1999. 